\title{
Du nouveau dans le syndrome de grêle court ? - Introduction
}

\author{
F. Joly $\cdot$ Y. Panis \\ (C) Lavoisier SAS 2016
}

Cette année est une grande année pour le monde de la nutrition et de l'insuffisance intestinale chronique. En effet, des recommandations européennes ont été publiées, mettant sur le devant de la scène ces pathologies rares et complexes qui nécessitent une prise en charge dans les centres experts. Le syndrome de grêle court (SGC) est en effet une de ces maladies rares qui nécessitent une prise en charge multidisciplinaire tout au long de son évolution, de la phase opératoire jusqu'au retour à domicile. Les acteurs sont multiples et il est parfois difficile de trouver l'interlocuteur qui permettra d'aider à prendre les décisions de réanimation, de modalités opératoires et le type de chirurgie nécessaire ainsi que la prise en charge nutritionnelle. La cause principale de SGC en France est l'infarctus mésentérique. Si pendant longtemps, cette pathologie était synonyme d'une mortalité très élevée, voire d'un renoncement à une prise en charge médicochirurgicale agressive, aujourd'hui, il est essentiel de prendre conscience que la survie est bonne lorsque la prise en charge est optimale et que cette prise en charge doit débuter dès la suspicion d'ischémie pour tenter d'éviter les résections étendues. Nous verrons ainsi par cette revue les différents aspects de la prise en charge du SGC, de la phase précoce à la phase stable, où dans un certain nombre de cas, malgré une prise en charge adéquate, le patient reste dépendant d'une nutrition parentérale à domicile parfois pour la vie. De plus, de nouveaux traitements médicamenteux sont disponibles en France pour améliorer l'absorption intestinale résiduelle du patient avec SGC, ce qui change totalement les perspectives au long cours pour les patients, aussi bien en termes de dépendance à la nutrition parentérale qu'en termes de qualité de vie. 